\title{
Development of a Robotic Assembly for Analyzing the Instantaneous Axis of Rotation of the Foot Ankle Complex
}

\author{
Kelly N. Salb, Daniel M. Wido, Thomas E. Stewart, and Denis J. DiAngelo \\ BioRobotics Laboratory, Department of Orthopaedic Surgery and Biomedical Engineering, \\ The University of Tennessee Health Science Center, 956 Court Avenue, Suite E226, Memphis, TN 38163, USA
}

Correspondence should be addressed to Denis J. DiAngelo; ddiangelo@uthsc.edu

Received 14 September 2015; Accepted 16 February 2016

Academic Editor: Kiros Karamanidis

Copyright (C) 2016 Kelly N. Salb et al. This is an open access article distributed under the Creative Commons Attribution License, which permits unrestricted use, distribution, and reproduction in any medium, provided the original work is properly cited.

\begin{abstract}
Ankle instantaneous axis of rotation (IAR) measurements represent a more complete parameter for characterizing joint motion. However, few studies have implemented this measurement to study normal, injured, or pathological foot ankle biomechanics. A novel testing protocol was developed to simulate aspects of in vivo foot ankle mechanics during mid-stance gait in a human cadaveric specimen. A lower leg was mounted in a robotic testing platform with the tibia upright and foot flat on the baseplate. Axial tibia loads (ATLs) were controlled as a function of a vertical ground reaction force (vGRF) set at half body weight ( $356 \mathrm{~N}$ ) and a $50 \%$ vGRF $(178 \mathrm{~N})$ Achilles tendon load. Two specimens were repetitively loaded over 10 degrees of dorsiflexion and 20 degrees of plantar flexion. Platform axes were controlled within 2 microns and 0.008 degrees resulting in ATL measurements within $\pm 2 \mathrm{~N}$ of target conditions. Mean ATLs and IAR values were not significantly different between cycles of motion, but IAR values were significantly different between dorsiflexion and plantar flexion. A linear regression analysis showed no significant differences between slopes of plantar flexion paths. The customized robotic platform and advanced testing protocol produced repeatable and accurate measurements of the IAR, useful for assessing foot ankle biomechanics under different loading scenarios and foot conditions.
\end{abstract}

\section{Introduction}

The kinematic and structural properties of human joints may be affected by diseases, injuries, or surgical alterations. In the case of the ankle joint, any disease, injury, or elected surgery, like osteoarthritis or ankle arthroplasty, will impact both the motion behavior of the joint (how it moves) and its structural stiffness properties (how it rotates under a muscle load) [1-6]. In vivo studies [5, 7-11] and computational models on ankle biomechanics [12-15] typically analyze the range of motion (ROM) or joint stiffness properties. Joint motion can also be described by rolling and sliding of articular surfaces during motion representative of a moving axis of rotation with dependency on loading scenarios [12]. The two-dimensional instantaneous axis of rotation (IAR) represents a more advanced parameter for characterizing joint kinematics, yet it has been void from most ankle biomechanics research. The ability to detect shifts in IAR may help in defining injury type and/or the impact of injury on foot ankle mechanics, as well as the effects of surgical procedures and implant and orthotic design.

Some protocols assume that the ankle complex behaves like a hinge joint with a single axis of rotation, whereas other studies suggested that a fixed axis of rotation with articular congruence may be an incorrect kinematic description for ankle joint motion [8, 12-17]. Few biomechanical testing platforms offer a physiologic loading environment [18-21]: some only investigate one instance of gait $[18,22]$, and others apply loads or force a kinematic profile estimated by a single specimen $[18,20,23]$.

To date, few studies have been conducted which address a two-dimensional instantaneous axis of rotation (IAR) or three-dimensional instantaneous helical axis (IHA) analysis of the ankle joint $[7,9,24]$. IAR data provide additional parameters for characterizing tibiotalar joint motion, where measurements are a direct representation of the effects of joint articular geometry and soft tissue structures. Threedimensional gait studies have demonstrated minimal motion 
out of the sagittal plane during stance phase gait, reducing the need for analyses of ankle joint motion to two dimensions (i.e., IAR). Quantifying the IAR of the ankle joint during gait may have the potential to advance the understanding of the biomechanical properties of the foot and ankle, including arch formation, ankle arthroplasty design, and surgical technique, and effects of orthotics and footwear.

Current gait simulators and biomechanical testing protocols either are unable to analyze the IAR, chose not to include this parameter as part of the analysis [4, 16, 18-23, 25-29], or are limited to high errors in the calculation of the IAR due to methodology $[7,24,29]$. There is a need for a biomechanical testing platform and protocol that will provide simulation of controlled tibia and Achilles tendon (AT) loads without constraining the foot ankle kinematic profile.

The purpose of this work was to establish a $2 \mathrm{D}$ testing method to determine the location of the IAR of the ankle joint under simulated stance phase conditions in a human cadaveric model. Additional tests were done to confirm the accuracy and repeatability of the IAR measurement. Once the rotational axis is known (describing where the ankle motion occurs), a more in-depth analysis of the ankle joint's mechanics can be undertaken (such as converting the external joint moment into its internal components of a muscle force times a moment arm), a level of in vitro analysis currently not possible with current testing methods. Further analysis of the path of the ankle joint's rotational axis offers new insight into the effects that disease and surgical alterations have on the ankle kinematics and mechanics.

\section{Materials and Methods}

2.1. Specimen Preparation. A matched pair (male, age of 37 and body weight of $712 \mathrm{~N}$ ) of human below knee lower extremity specimens was procured from Restore Life USA (Johnson City, TN). Specimens were frozen at $-20^{\circ} \mathrm{C}$. Before preparation, the feet were thawed in a refrigeration system for two days. Medial and lateral radiographs were used to verify the absence of anatomical abnormalities or surgery. Soft tissue and muscle were resected to expose approximately $100 \mathrm{~mm}$ of the proximal shafts of the tibia and fibula. A single one inch \#6 wood screw was then placed across the proximal ends of the tibia and fibula to stabilize the bones and add additional fixation for the potting material. After the AT was exposed and soft tissue was excised to allow approximately $150 \mathrm{~mm}$ of AT length for clamping, a cable puller was attached and secured with a U-bolt to increase clamping power. The tibial shafts were then mounted concentrically into a cylindrical pot using an alignment frame to position the tibia in a neutral vertical orientation. Low-melting-point bismuth alloy (Rotometals Inc., San Leandro, CA) was used to fix the tibia and fibula together and create a mounting fixture for attachment to the robotic testing platform (RTP). The final prepared specimen is shown in Figure 1.

2.2. Robotic Testing Platform. An existing custom designed, multiaxis testing platform was utilized to simulate aspects of stance phase gait mechanics under displacement control and force feedback (Figure 2) [30]. Two linear actuators, a

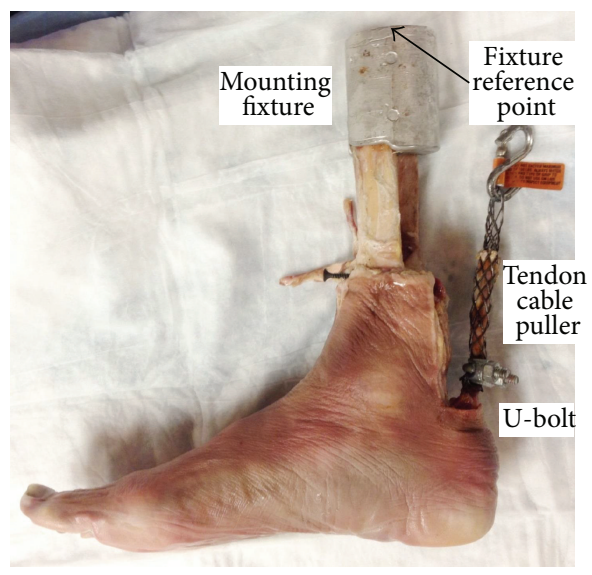

FIgURE 1: A prepared below the knee lower extremity specimen. Tissue was resected from the proximal shafts of the tibia, fibula, and AT. A cable puller was attached to the AT for tendon load application. Bismuth alloy material was used to create a mounting fixture for the proximal shafts of the tibia and fibula for rigid fixation to the testing platform. The fixture reference point was located at the center of the upper mounting fixture.

Parker Hannifin Corp. (Cleveland, OH) 406XR series linear ball screw actuator and an Exlar (Chanhassen, MN) GSX30 linear roller screw actuator were aligned in $x$-axis and $z$ axis, respectively. Control specifications of platform actuators were 2 microns in $X$ and 0.31 microns in $Z$. The rotary motors and drive units of the original test RTP were upgraded with Harmonic Drive units (Peabody, MA: model FHA-25C160-US250) having improved resolutions of 0.008 degrees. Previous position control pilot trials demonstrated that each individual axis was controllable to within tolerance of \pm 2 times the resolution of each feedback sensor corresponding to \pm 0.0091 degrees, \pm 4 microns in $z$ direction, and \pm 0.6 microns in $x$ direction for sagittal plane movement.

Two six-axis load cells (JR3 Inc., Woodland, CA: models $100 \mathrm{M} 40$ and $45 \mathrm{E} 15 \mathrm{~S}$ ) were attached to the robotic testing platform assembly and measured the three orthogonal forces and moments applied to the tibia via the gimbal assembly and forces transferred through the foot to the ground at the base of an $X-Y$ table. Load cell resolutions and capacities were $800 \pm 0.44 \mathrm{~N}$ in $z$-axis, $400 \pm 0.09 \mathrm{~N}$ in $x$-axis and $y$-axis, and $40 \pm 0.02 \mathrm{Nm}$ about all axes, respectively. The load cell rated accuracies were $1.0 \%$ of full scale with linearity error of $0.1 \%$ full scale. The robot testing system, actuator fixtures, and load cells were significantly greater in stiffness than those of human tissue, such that any deflection under test loading was negligible (i.e., less than 50 microns for $1000 \mathrm{~N}$ load). All reported deformation represented the compliancy of specimen tissue.

Potted specimens were clamped securely in a mounting block and rigidly connected to the testing platform. A sagittal plane was established for each specimen by bisecting the second metatarsal and AT [11, 13,17] with a vertical axis aligned with the tibia that matched the $X-Z$ plane of the testing platform. A static AT force vector $\left(F_{a}\right)$ was applied via a cable-pulley system designed to minimize friction to 


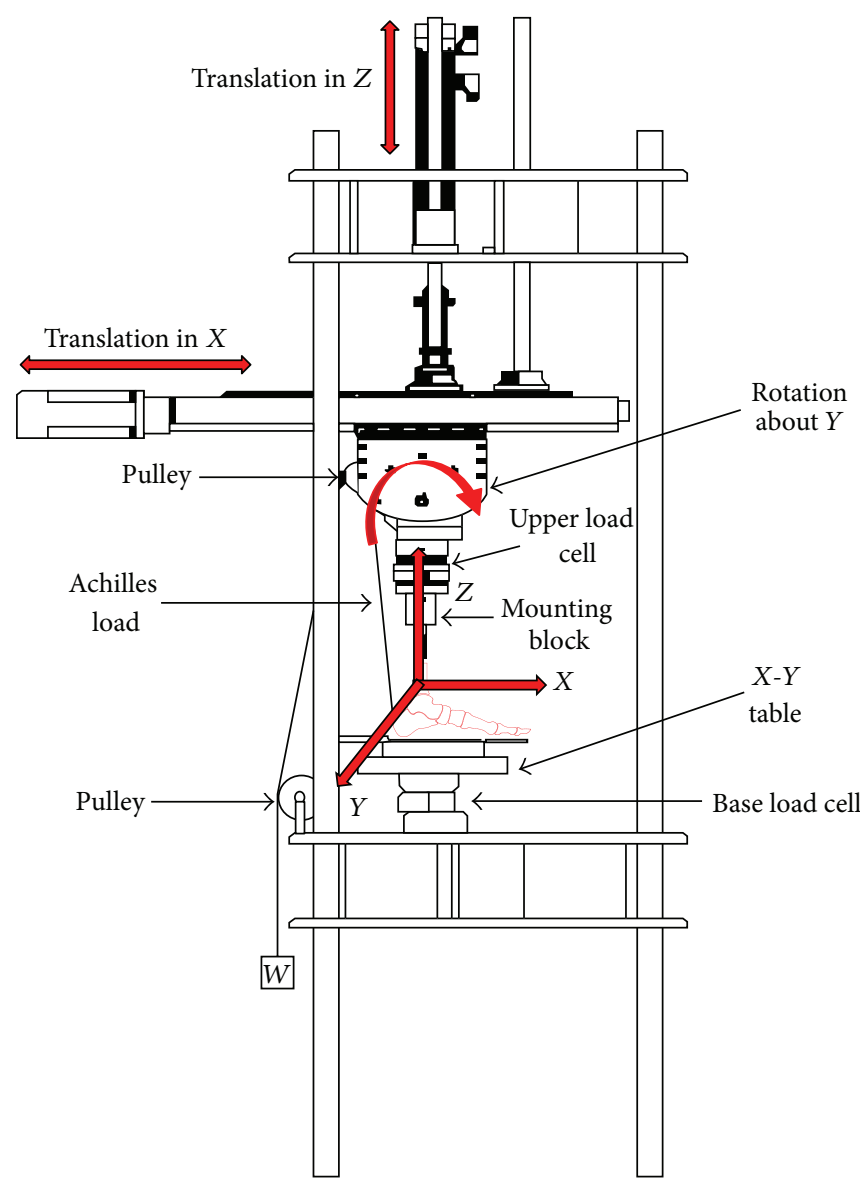

FIGURE 2: Diagram of multiaxis robotic system configured for the ankle study [30]. The testing platform consisted of an upper gimbal assembly with two translational and one rotational axes along with a 6-axis load cell, a mounting block to rigidly affix the specimen to the RTP, a pulley system to apply a static load to the AT, and an $X-Y$ table with a second 6-axis load cell to aid in the mounting procedure. The coordinate system is shown by $x$-axis, $y$-axis, and $z$-axis. Translational and rotational vectors of the controlled axes of the RTP are also shown.

within four degrees of the vertical tibia axis, while the heel was raised approximately $50 \mathrm{~mm}$ and the $X-Y$ table was unlocked (Figure 3). This feature allowed for unconstrained arch formation while transferring loads from the tibia to the calcaneus and maintaining foot support throughout the gait simulation $[31,32]$. The heel was then returned to the neutral position and an axial tibia force $\left(F_{t}\right)$ was applied to the specimen to meet the desired vertical ground reaction force (vGRF) condition within a prescribed $\pm 2 \mathrm{~N}$ tolerance. After the desired loading condition was met, the $X-Y$ table was locked and the rotation of the specimen was started.

2.3. Test Protocol. Custom software (Adept Inc., San Jose, CA) was written to simulate the two-dimensional biomechanics of the ankle joint during the early stance phase of walking gait. A rigid body analysis was used to describe the dynamic loading characteristics of the ankle as a function of an Achilles load and the external joint reaction forces $\left(F_{x}, F_{z}\right)$ and bending moment $(M)$. The relationship between the AT force $\left(F_{a}\right)$, the tibia force, and the vertical ground reaction force (i.e., vGRF) minus the weight of the foot is shown in Figure 4. Using this relationship, the AT was statically loaded as a percentage of the vGRF acting on the foot and ankle set at half body weight $(356 \mathrm{~N})$ and a $50 \%$ vGRF $(178 \mathrm{~N})$ AT load was applied based upon values of physiologic peak contractive tension of the AT $[9,11]$. These conditions were selected within the $800 \mathrm{~N}$ capacity of the upper load cell to achieve reproducible, subinjurious loading conditions.

With the potted specimen mounted and aligned to the testing platform (Figure 5), the fixture reference point located on the upper mounting fixture (Figure 5(a)) was transferred to the null tool tip (NTT) of the robot located at the center of the gimbal assembly. From there, a tool tip transformation was applied to reposition the robot's rotational tool tip axis to the initial prescribed ankle rotational axis (pARA) (located at the center of the talus) which was measured off of a scaled medial radiograph of the foot processed in ImageJ (NIH).

The tibia started in a neutral orientation ( 0 degrees) and was rotated about the initial pARA (Figure 5(a)). After each rotation, the position of the pARA was changed a small amount (usually between 50 and 100 microns) along either direction of the rotated $x$-axis and $z$-axis so as to reduce the forces acting along those axes and the associated incremental moment contribution $\left(\Delta M_{\text {pARA }}\right)$. By reducing these off-axis forces to $2 \mathrm{~N}$ or less, the only load responsible for creating the joint rotation was the applied moment (i.e., 


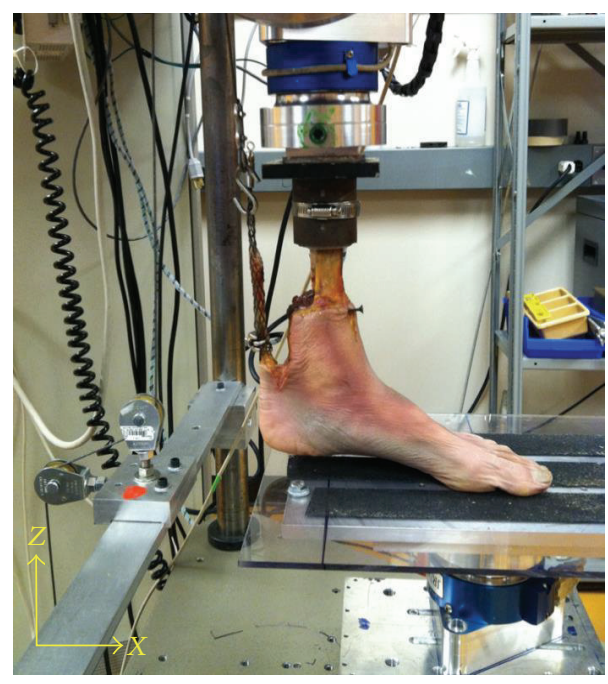

FIgURE 3: Specimen mounted in the RTP. This image shows clearance between the heel and $X-Y$ table as the AT load is applied, allowing unconstrained arch formation.

$\left.\Delta M_{\text {pARA }} \sim 0\right)$. This new location was considered as the true ARA (Figure 5(b)) and represented a "pure ankle moment" loading condition.

Once the pure moment condition was established, the shear and compressive forces $\left(F_{x}^{\prime}\right.$ and $F_{z}^{\prime}$ in Figure 6) were added to maintain the axial tibial force and vGRFs. Furthermore, by applying these forces along the new rotational axes that pass through the ankle rotational axis, the applied pure ankle moment condition was not altered. The process was repeated every 0.5 degrees until the full 10 degrees of dorsiflexion or 20 degrees of plantar flexion were achieved. A unique feature of the robotic testing platform was the ability to play back the motion and loading conditions in real time. Throughout the testing sequence, specimens were kept moist at regular intervals with a $0.9 \%$ saline mist.

2.4. Data Analysis. Force and positional data were sampled at $20 \mathrm{~Hz}$. A modified set of equations originally derived by Crisco III et al. [33] were applied to the NTT rotational and positional data for each increment of rotation to calculate the IAR of the ankle in the sagittal plane. Unlike previous studies that used positional data from camera targets or radiographs to calculate the IAR $[7,24]$, this study used the positional data from the robot to determine the IAR values. In doing so, the overall error in the IAR calculations of the entire testing system (including the testing frame, actuator, gimbal assembly, mounting fixtures, and load cell) was within $\pm 0.050 \mathrm{~mm}$ for loads up to $1000 \mathrm{~N}$. The path of the IAR was plotted back onto the specimen and expressed relative to an "ankle coordinate frame" that was defined by the bisector of the tibia and highest point on the tibial mortise in the initial neutral vertical orientation (Figure 7). The same process used to determine the location of the pARA and fixture reference point (Figure 2) was used to locate the ankle coordinate frame.

The test protocol cycled each specimen five times for tissue conditioning and five times in dorsiflexion and plantar
TABLE 1: Average axial tibia loading conditions.

\begin{tabular}{lcc}
\hline Specimen & \multicolumn{2}{c}{$\begin{array}{c}\text { Axial tibia load (N) } \\
\text { (Mean } \pm \text { standard deviation) }\end{array}$} \\
\hline 1 & Dorsiflexion & Plantar flexion \\
2 & $516.5 \pm 1.0$ & $506.0 \pm 1.5$ \\
\hline
\end{tabular}

flexion to demonstrate repeatability. The final three cycles were plotted and analyzed statistically using GraphPad Prism 6.0 software (La Jolla, CA). Mean axial tibia forces and mean $X$ IAR values and $Z$ IAR values were compared separately for dorsiflexion and plantar flexion using One-Way ANOVA with Holm-Š́dák post hoc test for multiple comparisons ( $\alpha=0.05$ ) to show variability between cycles. The mean $X$ IAR values and $Z$ IAR values for all three cycles were also compared between dorsiflexion and plantar flexion. Additionally, the slopes of the plantar flexion IAR paths were compared between cycles using a linear regression analysis in GraphPad Prism 6.0 software. Results are presented as means \pm standard deviations. A $P$ value of less than 0.05 signified significant differences between comparisons.

\section{Results}

The mean axial tibia loads throughout full rotation for each specimen are shown in Table 1. No statistical differences were found between cycles of motion $(P>0.05$ for all comparisons). The maximum tibial force error was less than $1 \%$ of the targeted value during dorsiflexion and less than 3\% during plantar flexion.

Mean IAR values for specimen 1 and specimen 2 are provided in Tables 2 and 3, respectively. No statistical differences were found in mean $X$ IAR values and $Z$ IAR values between cycles of dorsiflexion or plantar flexion for either specimen ( $P>0.05$ for all comparisons). When comparing mean IAR values for all three cycles between dorsiflexion and plantar flexion, statistically significant values were found in the mean $X$ IAR values for both specimens $(P<0.0001)$ and in the mean $Z$ IAR values in specimen $2(P<0.0001)$.

The final 3 cycles of IAR pathways are plotted relative to the ankle coordinate frame for specimen 1 (Figure 8) and specimen 2 (Figure 9) along with mean IAR values and standard deviations. The IAR represents a moving axis of rotation due to the joint articular geometry and surrounding soft tissue structures. Dorsiflexion paths show little movement in the talus, remaining within a $4.5 \mathrm{~mm}$ range (i.e., $2 \mathrm{~mm}$ in $X$ and $4 \mathrm{~mm}$ in $Z$ ). In plantar flexion, the first degrees of motion started up within the articular surface of the talus. As rotation continued, the IAR path moved downward towards the middle of the talus. The plantar flexion IAR path had a maximum range of approximately $21 \mathrm{~mm}$ (i.e., $20 \mathrm{~mm}$ in $Z$ and $6 \mathrm{~mm}$ in $X$ ). A linear regression analysis was performed on the plantar flexion paths. The slopes of the IAR paths for dorsiflexion testing are listed in Table 4. No statistical differences were found between the slopes for specimen 1 $(P=0.7823)$ or specimen $2(P=0.0826)$. 


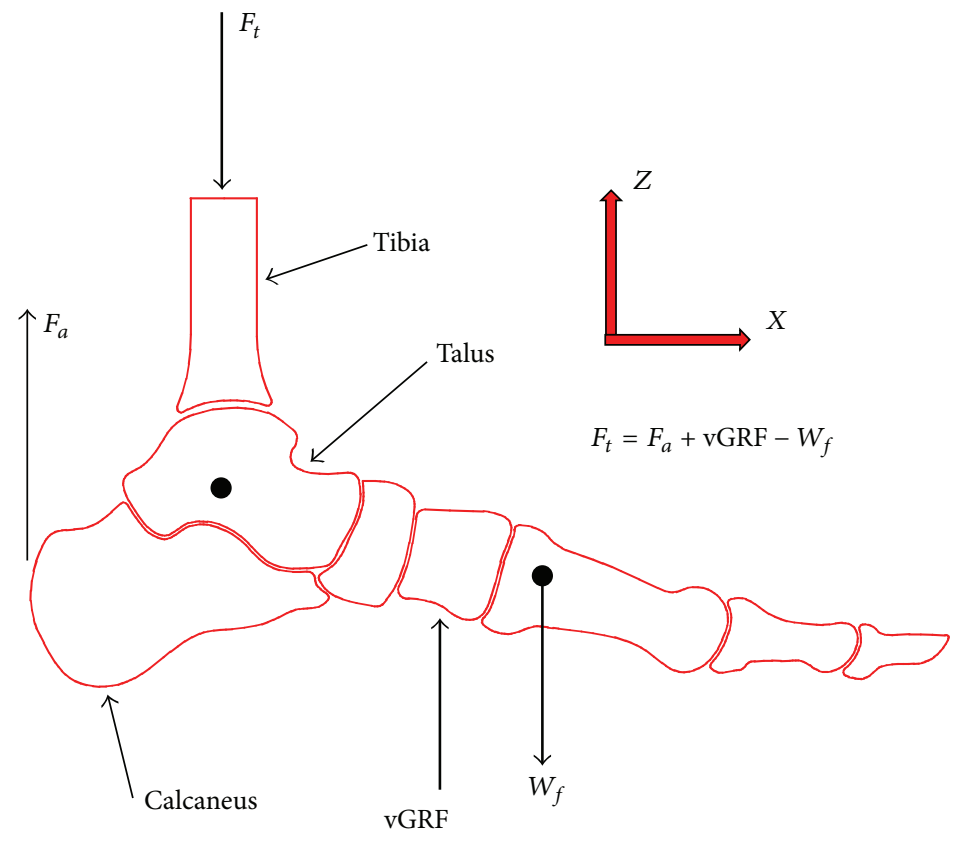

FIGURE 4: Force analysis of the foot and ankle used to set the parameters of loading, where $F_{t}$ represented the axial tibia load, $F_{a}$ was the AT load, vGRF was the vertical ground reaction force, and $W_{f}$ was the weight of the foot and tibia.

TABLE 2: Mean IAR values for specimen 1.

\begin{tabular}{lcccc}
\hline Cycle & \multicolumn{2}{c}{$\begin{array}{c}\text { XIAR }(\mathrm{mm}) \\
\text { (Mean } \pm \text { standard deviation) }\end{array}$} & \multicolumn{2}{c}{$\begin{array}{c}\text { ZIAR (mm) } \\
\text { (Mean } \pm \text { standard deviation) }\end{array}$} \\
& Dorsiflexion & Plantar flexion & Dorsiflexion & $-8.85 \pm 5.20$ \\
1 & $-2.95 \pm 0.10$ & $1.00 \pm 1.30$ & $-9.00 \pm 0.50$ & $-9.30 \pm 4.70$ \\
2 & $-2.90 \pm 0.15$ & $0.55 \pm 1.10$ & $-9.40 \pm 0.45$ & $-8.70 \pm 5.40$ \\
\hline Mean & $-2.90 \pm 0.10$ & $1.05 \pm 1.30$ & $-9.20 \pm 0.55$ & $-8.95 \pm 0.30$ \\
\hline
\end{tabular}

${ }^{*}$ Statistically significant between dorsiflexion and plantar flexion mean IAR values.

\section{Discussion}

A novel dynamic robotic testing platform and protocol were developed and used to investigate the biomechanical behavior of the foot and ankle under simulated loading conditions that are representative of mid-stance gait. The first objective of this study was to validate the accuracy of a loading protocol. The protocol simulates a static Achilles load during early stance phase of walking with decreased in vivo loading conditions. The role of soft tissue in this protocol was accounted for by preconditioning of the cadaver feet by subjecting them to several load and movement cycles before relevant data were captured. The simulation accounted for 20 degrees of plantar flexion and 10 degrees of dorsiflexion, whereas the total functional range of walking motion of the ankle during stance phase is approximately 15 degrees $[4,5,11$, $34]$. Force errors were typically controlled within prescribed $\pm 2 \mathrm{~N}$ tolerances throughout testing. The ATLs were limited by the upper loading capacity of the gimbal load cell, such that loading conditions were set at half body weight $(356 \mathrm{~N})$ and the Achilles tendon force was set to half the vGRF $(178 \mathrm{~N})$. Nevertheless, the ground reaction forces produced during the simulation were in agreement with a percentage of recorded in vivo forces $[1,3,5,6,16,34]$. Likewise, for the simulated loading profiles, the applied tibial force remained within $3 \%$ of the targeted value.

The second objective of the current study was to address intraspecimen variability between cycles of motion. Stability of the ankle joint is determined by three main factors: articular congruity, ligamentous structures, and ankle position $[8,16]$. All have been shown to have high variability between specimens [35]. Baxter showed a difference in biomechanical properties of sprinters versus nonsprinters, suggesting that physical health plays a major role in mechanics of the ankle [7]. Therefore, the goal was to demonstrate a repeatable measurement of the IAR in the sagittal plane for each specimen. This loading protocol showed variability in the mean IAR measurements between cycles of motion (as measured by the standard deviation) of less than $1 \mathrm{~mm}$ in both specimens. For the specimens tested, the talus dimensions 


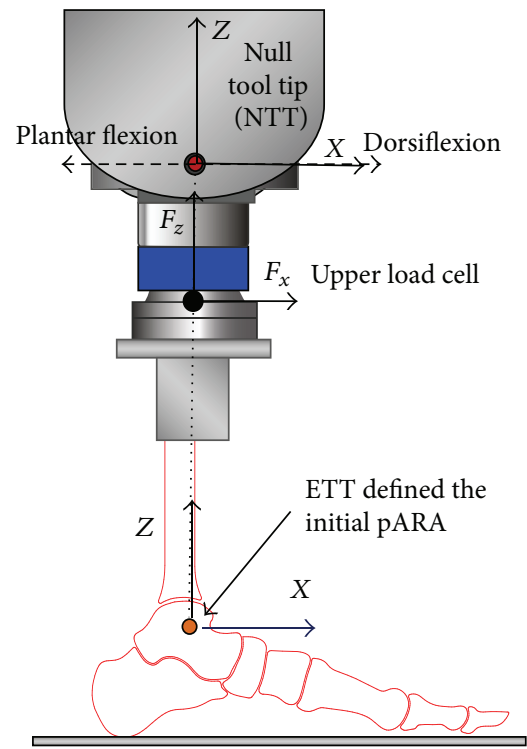

(a)

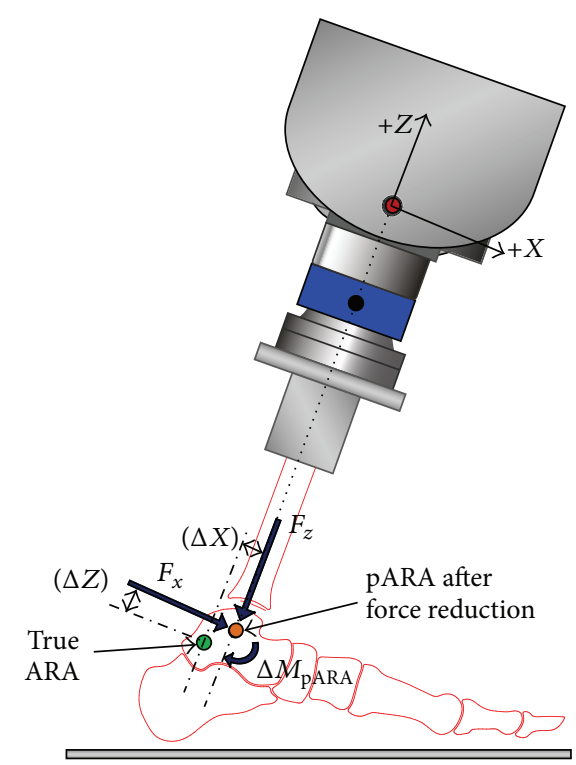

(b)

Figure 5: Kinematic and load control strategy. (a) Kinematic coordinate frames of the foot and ankle defined by the NTT and extended tool tip (ETT) of the RTP. The ETT (orange dot) represents the initial prescribed IAR of the foot located at the center of the talus and was a negative $Z$ offset from the NTT. The coordinate frame of the load cell was initially located at the center of the load cell and transformed to the origin of the moving joint center, that is, the ETT. (b) An angular input of ankle rotation was given every 0.5 degrees, during which the loading protocol reduced the ankle moment due to off-axis forces by minimizing the distance $(\Delta X, \Delta Z)$ between the pARA (upper) and the true ARA (lower), thus creating a "pure moment" condition.

TABLE 3: Mean IAR values for specimen 2.

\begin{tabular}{lcccr}
\hline \multirow{2}{*}{ Cycle } & \multicolumn{2}{c}{ XIAR $(\mathrm{mm})$} & \multicolumn{2}{c}{ ZIAR (mm) } \\
& Dorsiflexion & Plantar flexion & \multicolumn{2}{c}{ (Mean \pm standard deviation) } \\
\hline 1 & $-4.30 \pm 0.65$ & $-0.25 \pm 1.35$ & $-6.40 \pm 0.70$ & $-3.55 \pm 4.95$ \\
2 & $-4.30 \pm 0.50$ & $0.10 \pm 1.50$ & $-6.30 \pm 1.30$ & $-3.10 \pm 6.20$ \\
3 & $-4.30 \pm 0.25$ & $-0.10 \pm 1.50$ & $-6.70 \pm 0.55$ & $-3.60 \pm 5.30$ \\
\hline Mean & $-4.30 \pm 0.05^{*}$ & $-0.10 \pm 0.15^{*}$ & $-6.45 \pm 0.20^{*}$ & $-3.40 \pm 0.30^{*}$ \\
\hline
\end{tabular}

${ }^{*}$ Statistically significant between dorsiflexion and plantar flexion mean IAR values.

TABLE 4: Mean values of IAR slopes for specimen 1 and specimen 2 during plantar flexion.

\begin{tabular}{lcc}
\hline \multirow{2}{*}{ Cycle } & \multicolumn{2}{c}{ IAR slope values } \\
& Specimen 1 \pm standard deviation) \\
\hline 1 & $-4.0 \pm 0.2$ & Specimen 2 \\
2 & $-4.1 \pm 0.2$ & $-3.5 \pm 0.2$ \\
3 & $-4.1 \pm 0.2$ & $-3.9 \pm 0.2$ \\
Mean & $-4.0 \pm 0.1$ & $-3.5 \pm 0.2$ \\
\hline
\end{tabular}

were approximately $60 \mathrm{~mm}$ in width and $38 \mathrm{~mm}$ in height, demonstrating small relative variability on a clinical scale. While dorsiflexion paths remained within a $4.5 \mathrm{~mm}$ range, plantar flexion paths showed more movement. However, plantar flexion slopes were not significantly different between cycles of motion in specimen 1 or specimen 2, demonstrating a repeatable measurement of the IAR path.

Current radiographic practice for defining anatomical locations on a radiograph is $\pm 0.5 \mathrm{~mm}$ at best using advanced software programs like Medical Metrics Inc., 2012, and is more than double that amount when processed from standard radiographs. In this research a similar level of accuracy was introduced when selecting the initially prescribed ankle rotation axis (pARA). However, all subsequent measures of the change in the location of the rotational axis (i.e., the path of the IAR) were dependent on the resolution of the robot's controlled axes, which was $0.001 \mathrm{~mm}$. Collectively, the accuracy of the measured IAR calculated was \pm 50 microns. This was a significant improvement over current IAR measurements of the ankle joint, where high errors in the calculation of the IAR were reported due to test methodology $[7,24]$. Similar errors in determining the location of the IAR have been observed in other joint motion studies. For example, 


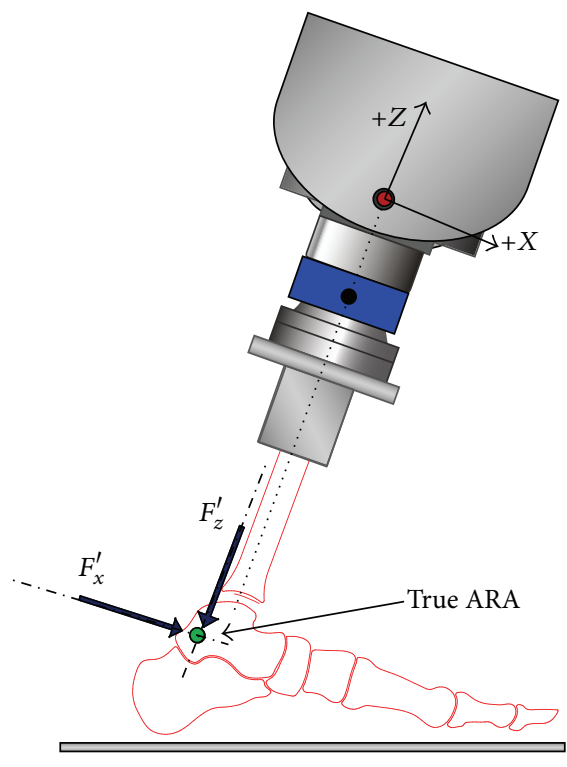

Figure 6: Kinematic and load control strategy for full load application simulation. Once the balanced position for pure moment loading was achieved (establishing the true ARA) the additional shear and compressive loads $\left(F_{x}^{\prime}, F_{z}^{\prime}\right)$ were introduced to maintain the axial tibial force and vGRFs. By passing these forces through the rotational axis of the ankle, the moment value was unchanged. The NTT data remained aligned with the rotated tibia axes. Achilles load is not shown.

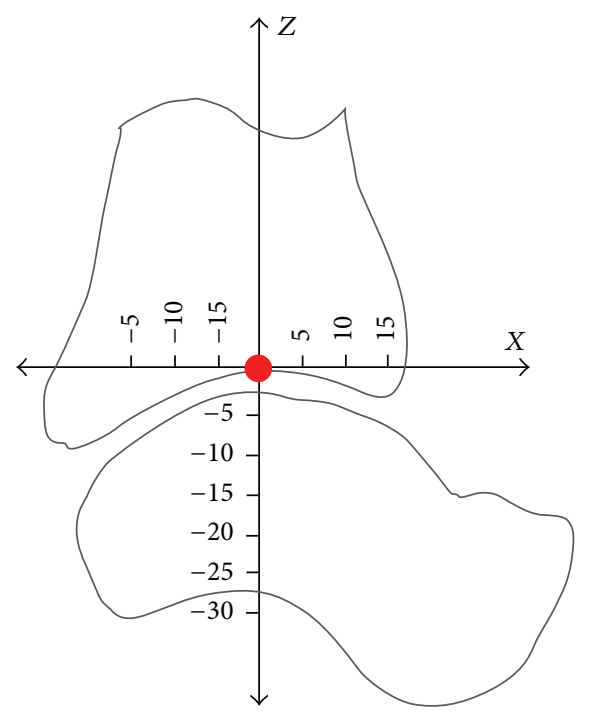

FIGURE 7: The "ankle coordinate frame" designated to plot the IAR on individual specimen. It was defined as the intersection of bisector of the tibia and the highest point on the tibial mortise.

in spinal motion studies, calculations of the IAR locations were greater than $20 \mathrm{~mm}$ for rotations less than 1 degree and approximately 3 to $4 \mathrm{~mm}$ for rotations up to 5 degrees [30].

Some limitations are present in this study. Only two specimens were studied. However, the goal of this study was to demonstrate minimal variability in the IAR measurement

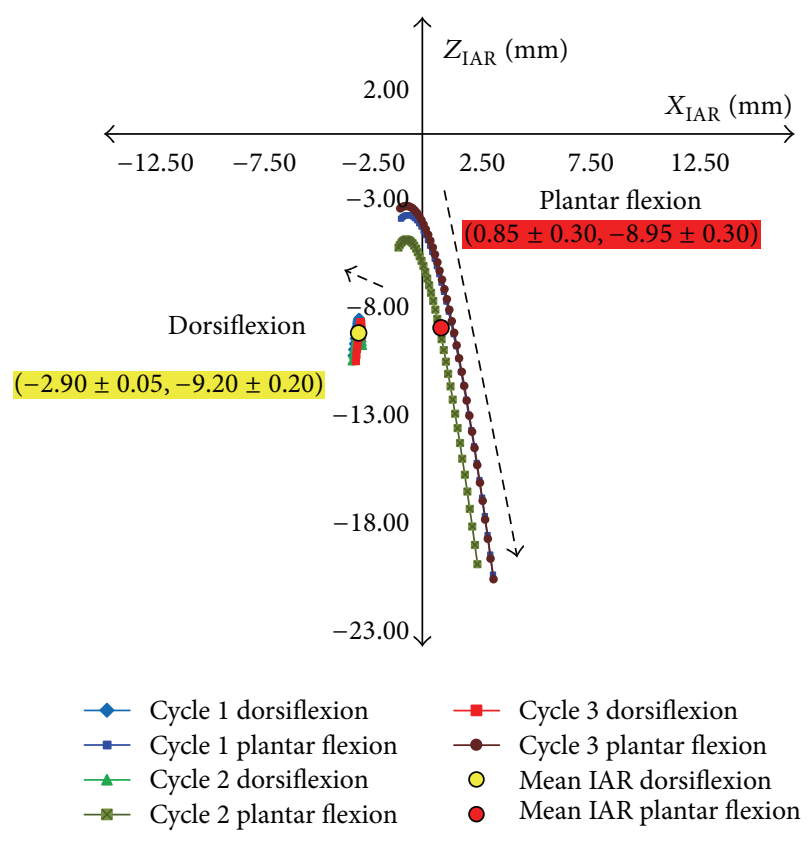

FIGURE 8: IAR paths of specimen 1 during dorsiflexion and plantar flexion plotted on medial radiographs. Mean IAR values for all three cycles (circles) with standard deviations (too small to see) are shown. Dashed arrows denote the direction of the IAR through rotation.

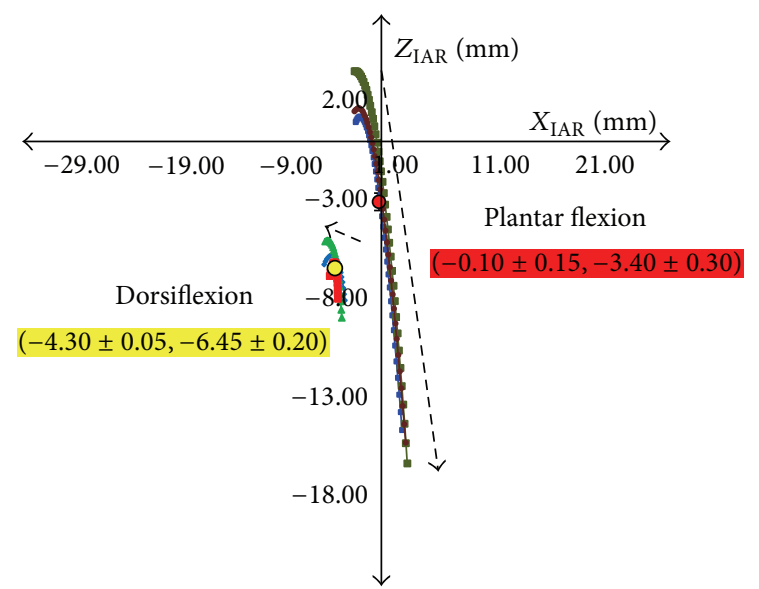

FIGURE 9: IAR paths of specimen 2 during dorsiflexion and plantar flexion plotted on medial radiographs. Mean IAR values for all three cycles (circles) with standard deviations (too small to see) are shown. Dashed arrows denote the direction of the IAR through rotation.

between cycles of motion in individual specimen. Additionally, the proximal fusion of the tibia and fibula removed the physiologic joint function where the bones may undergo translations and rotations relative to each other. This has been observed primarily when the knee experiences external and internal rotation [36]. Our protocol accounts for tibial sagittal plane motion only. During force application an outof-plane load was produced as a mode of lateral stabilization of the joint. However, a negligible moment build-up was observed throughout motion $(0.5 \mathrm{Nm})$ and did not restrict 
flexion. Additionally, the current study does not incorporate a dynamic Achilles tendon force profile representative of in vivo loading conditions, and it does not account for any of the other major plantar flexors, extrinsic dorsiflexors, or intrinsic muscles of the foot. However, these muscles are predominantly active during heel strike and heel rise to toe off gait $[8,34]$. The force applied via the Achilles tendon has been shown to have the greatest role in the biomechanical behavior of the ankle joint during stance phase when the foot is flat $[8,34]$. Therefore, it is the only tendon force accounted for in this study; no other intrinsic muscles were simulated. During normal gait, the tibia is driven from maximum plantar flexion to dorsiflexion with a continuous load on the joint. This protocol is limited in that the dorsiflexion and plantar flexion paths are generated independently. Furthermore, plantar flexion is driven in a direction uncharacteristic of the target in vivo stance phase motion. However, the Achilles tendon load is applied to the specimen in such a manner to simulate the in vivo loading scenario where the joint is plantar flexed. The independent movement from the neutral orientation between dorsiflexion and plantar flexion may explain the disconnection between the initial points of IAR paths in dorsiflexion and plantar flexion. Another limitation is that our study was two-dimensional, whereas the ankle joint can move in three dimensions $[1,16,29]$. While the talus and calcaneus were free to translate or rotate throughout motion, the IAR directly reflected the motion of the tibia defined by the robot tool tip which did not deflect from the sagittal plane. Motion during stance phase gait is typically limited to the sagittal plane with the exception of heel strike and heel rise $[11,16,37]$. Therefore, the IAR analysis could be limited to two dimensions. Increments of rotation could have been reduced to provide a more thorough and accurate IAR path. However, this was a very good compromise to describe the path within $5 \%$ of the total rotation.

\section{Conclusions}

This work has provided description of a novel loading protocol developed to support in vitro cadaveric testing of the foot and ankle complex. The protocol provided a repeatable measure of the two-dimensional analysis of the IAR of the ankle joint using tightly controlled loads in conjunction with a passive AT force. The low force error tolerance and consistent axial force values demonstrate the RTP's ability to accurately simulate forces. The results of the study are one of a handful to address the instantaneous axis of rotation of the ankle joint and provide the most accurate measurement to date. The IAR measurement was repeatable within one millimeter, smaller than what is feasibly measurable in a clinical setting. Because the IAR measurement is a direct representation of soft tissue structures and articular geometry, future in vitro studies may yield great insight into the biomechanical properties of the foot and ankle within the sagittal plane, including arch formation and effects of orthotics and footwear on ankle kinematics. Furthermore, much like in knee joint mechanics, where an injury to the ligaments within the joint can cause a shift in the IAR path of the knee, similar relationships may occur between the structures of the ankle and its IAR properties. Detection of a deviation in the IAR path can be associated with a specific injury mechanism and serve as a way to improve the corrective capacity of any treatment. As new information describing the dynamic loading characteristics of the foot and ankle is obtained from in vivo gait studies (stair climbing, orthotics, and footwear), this novel RTP and test protocol can readily simulate these conditions. Current development includes incorporating a dynamic Achilles tendon load, a higher capacity load cell to accommodate forces comparable to in vivo loads, and an optoelectronic motion tracking system to study the interaction of the bones of the arch of the foot throughout ankle rotation. Future use of the testing protocol and platform can be directed towards the study of other muscle groups on load and motion behavior of the foot ankle complex.

\section{Competing Interests}

The authors declare that they have no competing interests.

\section{Acknowledgments}

The authors would like to acknowledge Brian Kelly, Ph.D., and Richard Kasser, Ph.D., for their medical and technical contributions to the project.

\section{References}

[1] C. Bishop, G. Paul, and D. Thewlis, "Defining standards for modeling the biomechanics of the foot and ankle: a systematic review," Journal of Foot and Ankle Research, vol. 4, supplement 1, p. O9, 2011.

[2] N. Blitz, Contemporary Controversies in Foot and Ankle Surgery, An Issue of Clinics in Podiatric Medicine and Surgery, Elsevier, Philadelphia, Pa, USA, 1st edition, 2012.

[3] J. Hamill and K. M. Knutzen, Biomechanical Basis of Human Movement, Lippincott Williams \& Wilkins, New York, NY, USA, 2006.

[4] S. E. Jarrell, J. R. Owen, J. S. Wayne, and R. S. Adelaar, "Biomechanical comparison of screw versus plate/screw construct for talonavicular fusion," Foot \& Ankle International, vol. 30, no. 2, pp. 150-156, 2009.

[5] C. Kirtley, Clinical Gait Analysis: Theory and Practice, Elsevier, New York, NY, USA, 2006.

[6] M. Nordin and V. H. Frankel, Basic Biomechanics of the Musculoskeletal System, Lippincott Williams \& Wilkins, Philadelphia, $\mathrm{Pa}$, USA, 3rd edition, 2001.

[7] J. R. Baxter, T. A. Novack, H. van Werkhoven, D. R. Pennell, and S. J. Piazza, "Ankle joint mechanics and foot proportions differ between human sprinters and non-sprinters," Proceedings of the Royal Society B: Biological Sciences, vol. 279, no. 1735, pp. 20182024, 2012.

[8] A. S. Kelikian and S. Sarrafian, Sarrafian's Anatomy of the Foot and Ankle: Descriptive, Topographic, Functional, Lippincott Williams \& Wilkins, New York, NY, USA, 3rd edition, 2011.

[9] C. N. Maganaris, V. Baltzopoulos, and A. J. Sargeant, "Changes in Achilles tendon moment arm from rest to maximum isometric plantarflexion: in vivo observations in man," Journal of Physiology, vol. 510, no. 3, pp. 977-985, 1998.

[10] R. Semple, G. S. Murley, J. Woodburn, and D. E. Turner, "Tibialis posterior in health and disease: a review of structure 
and function with specific reference to electromyographic studies," Journal of Foot and Ankle Research, vol. 2, no. 1, article 24, 2009.

[11] D. A. Winter, Biomechanics and Motor Control of Human Movement, John Wiley \& Sons, Hoboken, NJ, USA, 4th edition, 2009.

[12] A. Leardini, J. J. O’Connor, F. Catani, and S. Giannini, "A geometric model of the human ankle joint," Journal of Biomechanics, vol. 32, no. 6, pp. 585-591, 1999.

[13] A. Leardini, J. J. O'Connor, F. Catani, and S. Giannini, "Kinematics of the human ankle complex in passive flexion; a single degree of freedom system," Journal of Biomechanics, vol. 32, no. 2, pp. 111-118, 1999.

[14] A. Leardini, "Geometry and mechanics of the human ankle complex and ankle prosthesis design," Clinical Biomechanics, vol. 16, no. 8, pp. 706-709, 2001.

[15] A. Leardini, J. J. O'Connor, F. Catani, and S. Giannini, "Mobility of the human ankle and the design of total ankle replacement," Clinical Orthopaedics and Related Research, vol. 424, pp. 39-46, 2004.

[16] M. D. Castro, "Ankle biomechanics," Foot and Ankle Clinics, vol. 7, no. 4, pp. 679-693, 2002.

[17] G. J. Sammarco, A. H. Burstein, and V. H. Frankel, "Biomechanics of the ankle: a kinematic study," Orthopedic Clinics of North America, vol. 4, no. 1, pp. 75-96, 1973.

[18] J. Burg, K. Peeters, T. Natsakis, G. Dereymaeker, J. V. Sloten, and I. Jonkers, "In vitro analysis of muscle activity illustrates mediolateral decoupling of hind and mid foot bone motion," Gait \& Posture, vol. 38, no. 1, pp. 56-61, 2013.

[19] K.-J. Kim, H. B. Kitaoka, Z.-P. Luo et al., "In vitro simulation of the stance phase in human gait," Journal of Musculoskeletal Research, vol. 5, no. 2, pp. 113-121, 2001.

[20] A. Suckel, O. Muller, P. Langenstein, T. Herberts, P. Reize, and N. Wulker, "Chopart's joint load during gait. In vitro study of 10 cadaver specimen in a dynamic model," Gait and Posture, vol. 27, no. 2, pp. 216-222, 2008.

[21] K. Watanabe, H. B. Kitaoka, T. Fujii et al., "Posterior tibial tendon dysfunction and flatfoot: analysis with simulated walking," Gait \& Posture, vol. 37, no. 2, pp. 264-268, 2013.

[22] R. Bahr, F. Pena, J. Shine, W. D. Lew, and L. Engebretsen, "Ligament force and joint motion in the intact ankle: a cadaveric study," Knee Surgery, Sports Traumatology, Arthroscopy, vol. 6, no. 2, pp. 115-121, 1998.

[23] N. A. Sharkey and A. J. Hamel, "A dynamic cadaver model of the stance phase of gait: performance characteristics and kinetic validation," Clinical Biomechanics, vol. 13, no. 6, pp. 420-433, 1998.

[24] T. Sumiya, Y. Suzuki, T. Kasahara, and H. Ogata, "Instantaneous centers of rotation in dorsi/plantar flexion movements of posterior-type plastic ankle-foot orthoses," Journal of Rehabilitation Research and Development, vol. 34, no. 3, pp. 279-285, 1997.

[25] C. Hurschler, J. Emmerich, and N. Wülker, "In vitro simulation of stance phase gait. Part I. Model verification," Foot \& Ankle International, vol. 24, no. 8, pp. 614-622, 2003.

[26] C. J. Nester, A. M. Liu, E. Ward et al., "In vitro study of foot kinematics using a dynamic walking cadaver model," Journal of Biomechanics, vol. 40, no. 9, pp. 1927-1937, 2007.

[27] L. D. Noble Jr., R. W. Colbrunn, D.-G. Lee, A. J. Van Den Bogert, and B. L. Davis, "Design and validation of a general purpose robotic testing system for musculoskeletal applications," Journal of Biomechanical Engineering, vol. 132, no. 2, pp. 1-12, 2010.
[28] E. C. Whittaker, P. M. Aubin, and W. R. Ledoux, "Foot bone kinematics as measured in a cadaveric robotic gait simulator," Gait \& Posture, vol. 33, no. 4, pp. 645-650, 2011.

[29] C. J. Nester, "Lessons from dynamic cadaver and invasive bone pin studies: do we know how the foot really moves during gait?" Journal of Foot and Ankle Research, vol. 2, article 18, 2009.

[30] B. P. Kelly and D. J. DiAngelo, "A multiaxis programmable robot for the study of multibody spine biomechanics using a real-time trajectory path modification force and displacement control strategy," Journal of Medical Devices, Transactions of the ASME, vol. 7, no. 3, Article ID 034502, 2013.

[31] D. B. Thordarson, H. Schmotzer, J. Chon, and J. Peters, "Dynamic support of the human longitudinal arch: a biomechanical evaluation," Clinical Orthopaedics and Related Research, no. 316, pp. 165-172, 1995.

[32] A. Aquino and C. Payne, "Function of the plantar fascia," The Foot, vol. 9, no. 2, pp. 73-78, 1999.

[33] J. J. Crisco III, X. Chen, M. M. Panjabi, and S. W. Wolfe, "Optimal marker placement for calculating the instantaneous center of rotation," Journal of Biomechanics, vol. 27, no. 9, pp. 1183-1187, 1994.

[34] M. M. Rodgers, "Dynamic biomechanics of the normal foot and ankle during walking and running," Physical Therapy, vol. 68, no. 12, pp. 1822-1832, 1988.

[35] R. P. Kleipool and L. Blankevoort, "The relation between geometry and function of the ankle joint complex: a biomechanical review," Knee Surgery, Sports Traumatology, Arthroscopy, vol. 18, no. 5, pp. 618-627, 2010.

[36] J. Scott, H. Lee, W. Barsoum, and A. J. Van Den Bogert, “The effect of tibiofemoral loading on proximal tibiofibular joint motion," Journal of Anatomy, vol. 211, no. 5, pp. 647-653, 2007.

[37] S. Yamaguchi, Y. Tanaka, S. Kosugi, Y. Takakura, T. Sasho, and S. A. Banks, "In vivo kinematics of two-component total ankle arthroplasty during non-weightbearing and weightbearing dorsiflexion/plantarflexion," Journal of Biomechanics, vol. 44, no. 6 , pp. 995-1000, 2011. 


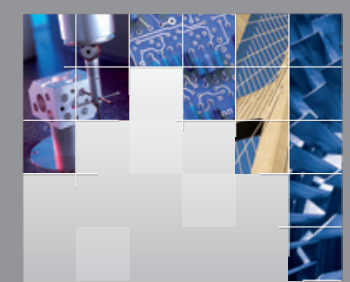

\section{Enfincering}
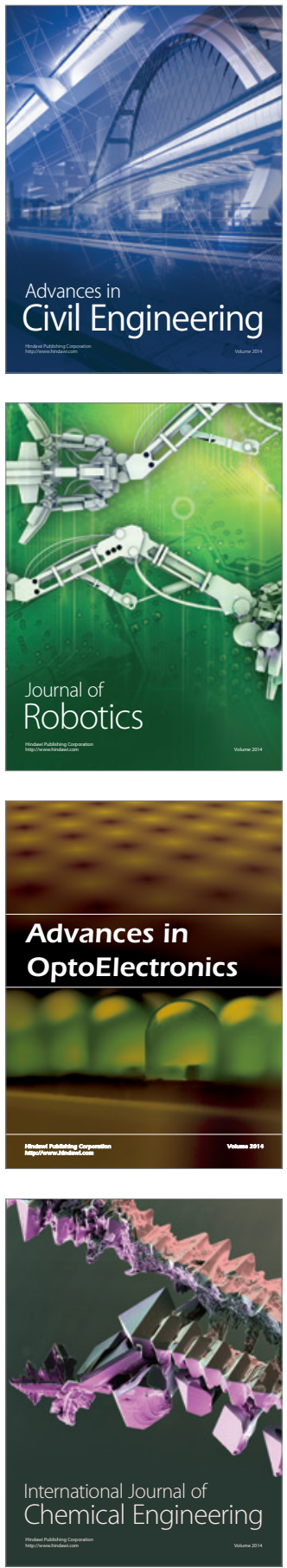

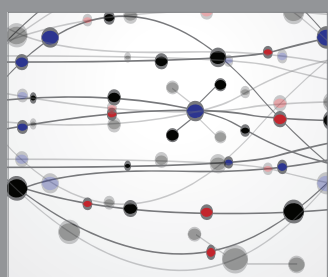

The Scientific World Journal

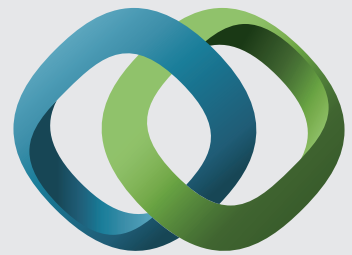

\section{Hindawi}

Submit your manuscripts at

http://www.hindawi.com
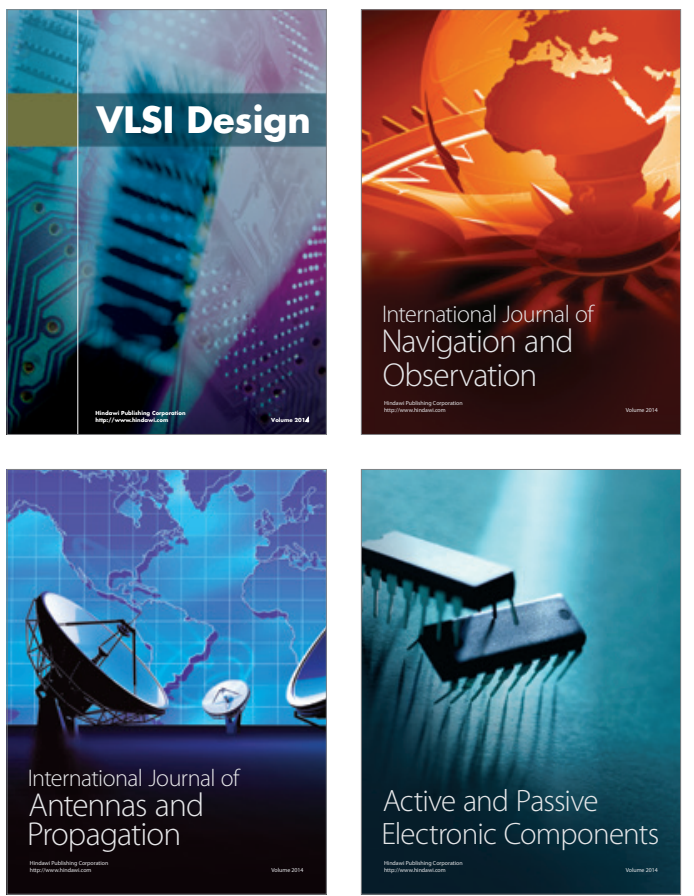
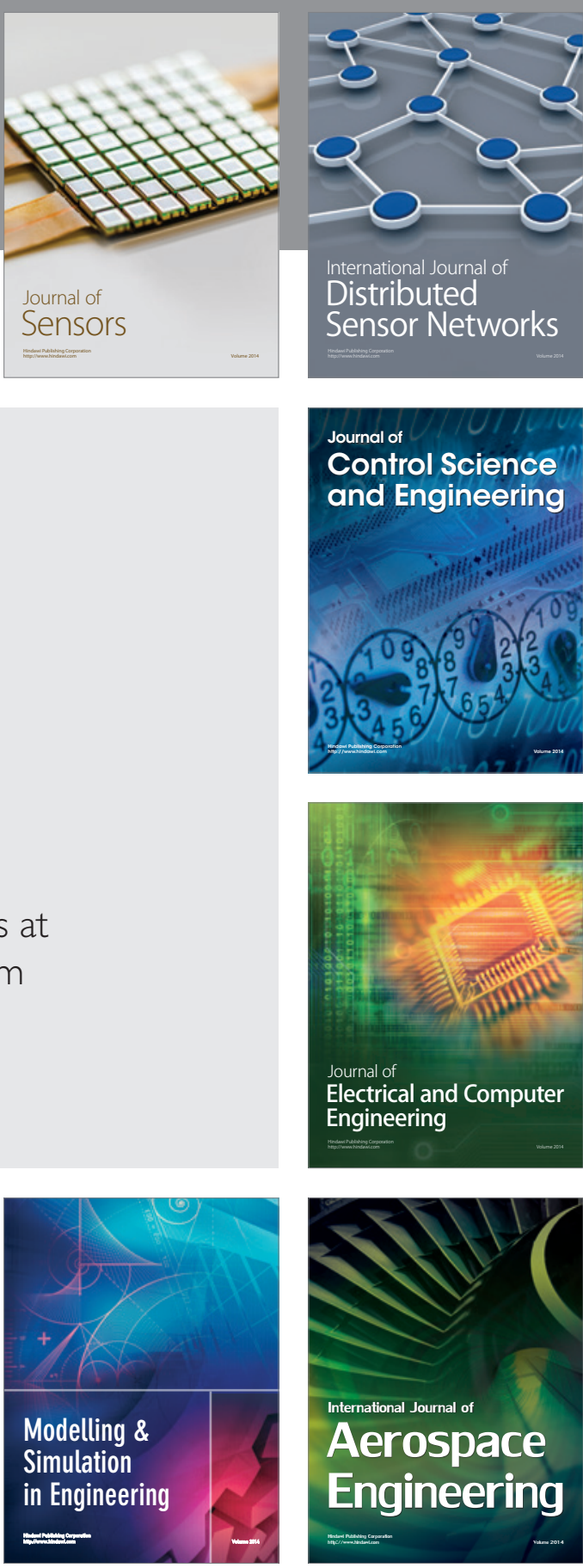

International Journal of

Distributed

Sensor Networks

Journal of

Control Science

and Engineering
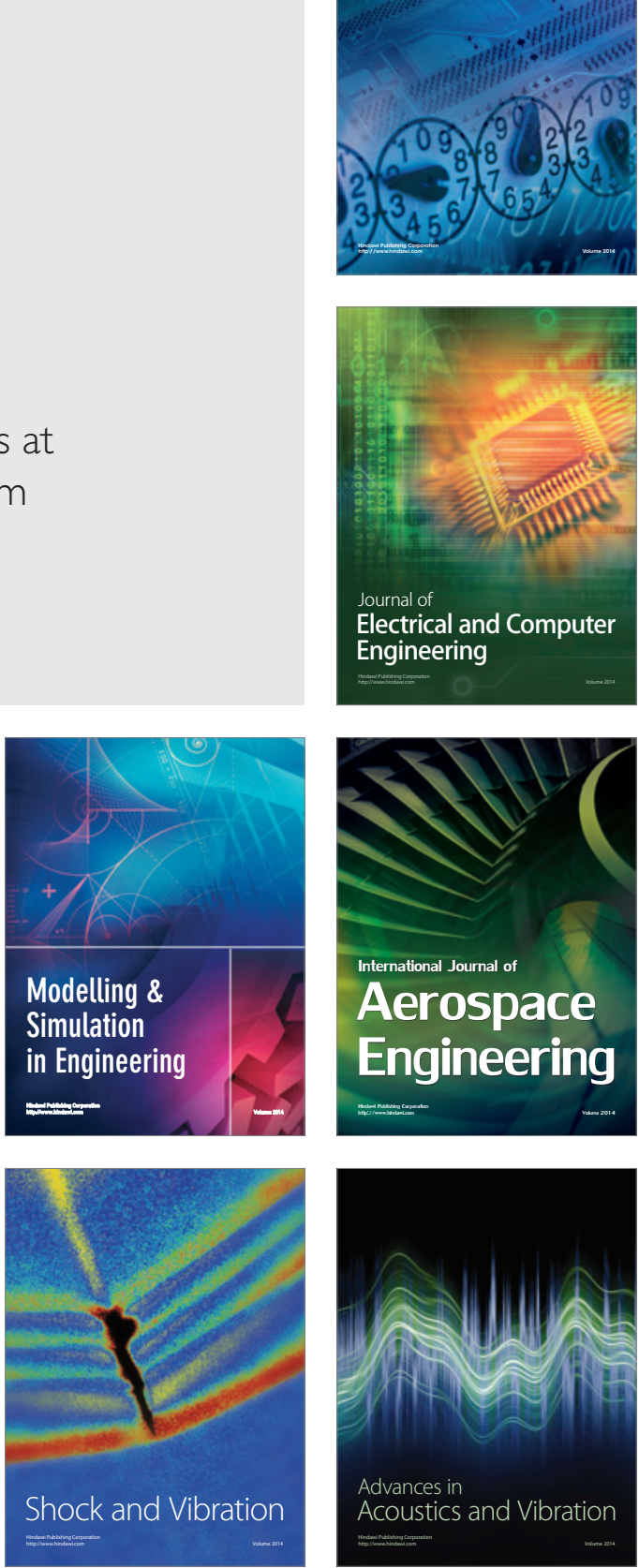